\title{
Elevated Serum Cyclophilin D Level is Associated with Nonalcoholic Fatty Liver Disease and Higher Fibrosis Scores in Patients with Diabetes Mellitus
}

\author{
Mervat Naguib' \\ Mahmoud Abou Elfotouh ${ }^{2}$ \\ Mohamed-Naguib Wifi ${ }^{2}$ \\ 'Diabetes and Endocrinology Unite, \\ Internal Medicine Department, Faculty of \\ Medicine, Kasr Al-Ainy Hospital, Cairo \\ University, Cairo, Egypt; ${ }^{2}$ Hepatology \\ Unite, Internal Medicine Department, \\ Faculty of Medicine, Kasr Al-Ainy \\ Hospital, Cairo University, Cairo, Egypt
}

Correspondence: Mervat Naguib Diabetes and Endocrinology Unite, Internal Medicine Department, Faculty of Medicine, Kasr Al-Ainy Hospital, 41 Manial Street, Cairo I I45I, Egypt

Tel +20 25729584

Fax +202628884

Email mervat.naguib@kasralainy.edu.eg
Background: Cyclophilin D (CypD) is a mitochondrial matrix protein involved in liver steatosis and fibrosis in vitro. However, the role of CypD in the development of fatty liver and liver fibrosis in humans has not been determined.

Purpose: To measure the serum level of CypD in patients with type 2 diabetes (T2DM) and nonalcoholic fatty liver disease (NAFLD) and to assess its relation to the presence of hepatic steatosis and fibrosis in this group of patients.

Patients and Methods: In this cross-sectional study, 30 patients with diabetes and NAFLD were compared to 30 patients with diabetes without NAFLD and 30 age- and sex-matched healthy subjects. Abdominal ultrasound was used to diagnose NAFLD. Serum CypD was measured using ELISA. Fibrosis-4 (FIB-4) index, AST to platelet ratio index (APRI), and NAFLD fibrosis score (NFS) were used as markers of liver fibrosis in patients with NAFLD. Patients with NAFLD were divided into two subgroups based on FIB-4 index: patients with liver fibrosis (FIB-4 >1.45) and patients without liver fibrosis (FIB-4 <1.45). CypD and other clinical and biochemical parameters were validated as predictors of NAFLD and liver fibrosis in diabetic patients in multivariate logistic regression analysis.

Results: Diabetic patients with NAFLD had higher serum CypD levels than those without NAFLD (11.65 \pm 2.96 vs $6.58 \pm 1.90 \mathrm{ng} / \mathrm{mL}$, respectively, $P<0.001)$. Correlation analysis revealed a significant positive correlation between CypD and FIB-4 index $(P=0.001)$, APRI $(P=0.013)$ and NFS $(P<0.001)$. GGT and CypD were the only predictors of NAFLD. For the prediction of significant fibrosis, AUROC of CypD was 0.835 with a cutoff $>14.05 \mathrm{ng} / \mathrm{mL}$ provides specificity of $81.8 \%$ and sensitivity of $75 \%$.

Conclusion: Serum CypD is related to hepatic steatosis and fibrosis in diabetic patients. Serum CypD may thus provide a novel marker and therapeutic target of NAFLD and liver fibrosis.

Keywords: cyclophilin D, fatty liver, fibrosis, type 2 diabetes

\section{Introduction}

Nonalcoholic fatty liver disease (NAFLD) is a major health problem worldwide. NAFLD affects up to $25 \%$ of the general population with highest prevalence among the Middle East population. ${ }^{1}$ NAFLD is usually recognized as the hepatic presentation of the metabolic syndrome. ${ }^{2}$ About $30 \%$ of patients with NAFLD were discovered to have type 2 diabetes mellitus (T2DM), and up to $80 \%$ of NAFLD cases have insulin resistance. ${ }^{3}$

The disease begins with hepatic steatosis and may advance to steatohepatitis that may progress to liver fibrosis and cirrhosis. ${ }^{4}$ Various factors are involved in the 
pathogenesis of NAFLD. Systemic insulin resistance is a key driver of hepatic steatosis in NAFLD. ${ }^{5}$ The initial insult of lipid excess is followed by multiple pathogenic processes including lipotoxicity, oxidative stress and immune system derangement. ${ }^{6}$

Mitochondrion is a key player in the metabolic homeostasis and mitochondrial dysfunction is related to hepatic insulin resistance and hepatic steatosis. ${ }^{7}$ NAFLD and diabetes are associated with changes in mitochondrial structure and impaired respiratory chain function leading to decreased adenosine triphosphate (ATP) synthesis, accumulation of triglycerides in hepatocytes and increased reactive oxygen levels. These changes lead to insulin resistance and hepatocyte inflammation that promotes cell death and development of fibrosis. ${ }^{8}$

Mitochondrial permeability transition pore (mPTP) is an important regulator of mitochondrial homeostasis and so the cellular metabolism. The mPTP extends between the outer and inner mitochondrial membranes and allows the passage of different molecules when it opens. ${ }^{9}$ Different stimuli like excess active oxygen species and increased calcium level promote opening of mPTP. ${ }^{10}$ Transient opening of $\mathrm{mPTP}$ is essential to maintain mitochondrial function, however, permanent opening of these channels under conditions of extreme cellular stress leads to the rapid passage of ions and large molecules that can lead to cell death. ${ }^{11}$

Cyclophilin D (CypD), is an $22 \mathrm{kDa}$ chaperone protein which is the product of the PPIF gene. CypD is a member of the cyclophilin family of peptidyl-prolyl, cis-trans isomerases (PPIases) that are present in the mitochondrial matrix. ${ }^{12}$ CypD has an important role in the regulation of mitochondrial function. CyPD controls the opening of mPTP to decrease ATP and increase production of reactive oxygen radicals. ${ }^{13}$ It was reported that CypD is related to ischemia reperfusion injury in the heart, lung, and kidney. ${ }^{13}$

Accumulating evidence suggest that CypD plays an important role in the development of hepatic steatosis and possibly will provide a new opportunity for the treatment of NAFLD. Increased CypD expression in hepatocytes stimulates mPTP excessive opening, subsequently causing endoplasmic reticulum stress and resulting in enhanced sterol regulatory element-binding protein-1c transcription and hepatic steatosis. ${ }^{14}$

However, CypD shows tissue-specific effects on metabolic homeostasis, for example, deletion of muscle CypD did not change the whole body glucose metabolism, while the loss of liver CypD was associated with development of obesity and altered the whole body metabolic dysfunction. ${ }^{12}$ Moreover, most of the previous studies were experimental and are based on the effect of CypD at mitochondrial level but no data about serum levels of this protein are available. ${ }^{13,14}$ The aim of this study was to investigate the serum level of CypD in diabetic patients with NAFLD and its relation to presence of hepatic fibrosis.

\section{Materials and Methods Study Design}

This study was a case-control study conducted in a tertiary care referral hospital between January 2017 and January 2018 after approval of the institutional ethical committee. We assigned 90 age and sex matched subjects included; 30 patients with T2DM and NAFLD, 30 patients with T2DM without NAFLD and 30 healthy controls. All subjects provided informed consent to participate in this study. The study protocol and procedures conform to the ethical guidelines of the 1975 Declaration of Helsinki. This study was approved by the Research Ethical Committee of Faculty of Medicine-Cairo University.

\section{Subjects and Methods}

Patients with diagnosis of T2DM based on ADA criteria were eligible for inclusion in this study. ${ }^{15}$ Exclusion criteria included patients with (1) diabetes other than T2DM; (2) chronic liver disease other than NAFLD; (3) history of alcohol intake or medications that might affect liver function; (4) history of cardiac or neurological diseases. Clinical data obtained included age, sex, waist circumference, BMI [weight $(\mathrm{kg}) /$ height $\left(\mathrm{m}^{2}\right)$ ], and duration of diabetes. Blood samples were collected from each individual after a period of 10-12 $\mathrm{h}$ fasting for measurement of fasting blood glucose (FBG), glycated hemoglobin (HbA1C), alanine aminotransferase (ALT), aspartate aminotransferase (AST), gammaglutamyl transpeptidase (GGT), international normalized ratio (INR), hemoglobin, platelet count, total cholesterol (TC), triglycerides (TG) and CypD.

Serum CypD level was measured using ELISA kit applies the competitive enzyme immunoassay technique utilizing a monoclonal anti-CypD antibody and an CypD-HRP conjugate (MyBioSource, Inc., CA, USA) according to manufacturer's instructions. Samples were collected in EDTA tubes; centrifugation was done for $15 \mathrm{~min}$ at $1000 \times \mathrm{g}$ at $2-$ $8^{\circ} \mathrm{C}$ within $30 \mathrm{~min}$ of collection and samples stored at $-80^{\circ} \mathrm{C}$. Before carrying out the test, serum samples were brought to room temperature and centrifuged. Absorbance 
measurements were carried out in a microplate reader at 450 $\mathrm{nm}$ and the concentrations were calculated using a standard curve.

Assessment of hepatic steatosis was done using abdominal ultrasound (US) after fasting overnight. All US scans were performed by the same sonographer using a high-resolution multifrequency B-mode scanner (SDD5500; Aloka, Tokyo, Japan) 2.5-5.0 MHz transducer. NAFLD was identified by the presence of at least two of the three following abnormal findings: diffusely increased echogenicity of the liver relative to the right kidney; attenuation of the ultrasound beam; poor visualization of intrahepatic architectural details. ${ }^{16}$

The fatty liver index score (FLI) was calculated in each participant using the following formula FLI =

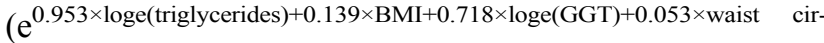
cumference-15.745 $) /\left(1+\mathrm{e}^{0.953 \times \operatorname{loge}(\text { triglycerides })+0.139 \times \mathrm{BMI}+0.718 \times \operatorname{loge}-}\right.$ $($ GGT $)+0.053 \times$ waist circumference-15.745 $) \times 100 .^{17}$ To assess liver fibrosis in patients with NAFLD fibrosis-4 (FIB-4) index was calculated using the formula: FIB-4=Age (years $) \times$ AST $(\mathrm{U} / \mathrm{L}) /[$ Platelet count $(109 / \mathrm{L}) \times$ ALT1/2 $(\mathrm{U} / \mathrm{L})] .{ }^{18}$ Diabetic patients with NAFLD were divided into two subgroups based on FIB-4 index: patients with liver fibrosis (FIB-4 $>1.45$ ) and patients without liver fibrosis (FIB-4 <1.45). The aspartate aminotransferases (AST) to platelet ratio index (APRI) score APRI $=($ AST in $\mathrm{IU} / \mathrm{L}) /(\mathrm{AST}$ upper limit of normal in IU/L)/(Platelets in $\left.10^{9} / \mathrm{L}\right)$ and NAFLD fibrosis score $(\mathrm{NFS})=-1.675+(0.037 *$ age $[$ years] $)+(0.094 * \mathrm{BMI}$ $[\mathrm{kg} / \mathrm{m} 2])+(1.13 * \mathrm{IFG} /$ diabetes $[$ yes $=1$, no $=0])+(0.99 * \mathrm{AST} /$ ALT ratio $)-(0.013 *$ platelet count $[\times 109 / \mathrm{L}])-(0.66 *$ albumin $[\mathrm{g} / \mathrm{dL}])$ were calculated in all patients with NAFLD. ${ }^{19,20}$

\section{Statistical Analysis}

Data was summarized using mean and standard deviation for quantitative variables and frequencies (number of cases) and relative frequencies (percentages) for categorical variables. Comparisons between groups were done using analysis of variance (ANOVA) with multiple comparisons post hoc test in normally distributed quantitative variables while nonparametric Kruskal-Wallis test and Mann-Whitney test were used for non-normally distributed quantitative variables. For comparing categorical data, chi-squared (v2) test was performed. Exact test was used instead when the expected frequency is less than five. Correlations between quantitative variables were done using Pearson's correlation coefficient. Univariate and multivariate logistic regression were done to detect independent predictors of fatty liver and fibrosis. Receiver operating characteristics (ROC) analysis was used to evaluate the diagnostic value of $\mathrm{CypD}$ to identify patients NAFLD and liver fibrosis. Area under the curve was generated using nonparametric assumption. Sensitivity and specificity values of CypD were profiled by curves. Data were coded and entered using the statistical package SPSS version 24. $P$-values less than 0.05 were considered as statistically significant.

\section{Results \\ Baseline Characteristics}

A total of 60 patients with T2DM (30 patients with NAFLD and 30 patients without NAFLD) and 30 healthy control subjects were enrolled in this study. By using ANOVA, there was no statistically significant difference between healthy control, diabetic patients without NAFLD and those with NAFLD regarding age (46.60 $\pm 9.61 ; 50.10 \pm 8.29 ; 50.10 \pm 8.45, P=0.212)$, BMI (28.66 $\pm 4.31 ; 28.48 \pm 5.40 ; 27.51 \pm 5.15, P=0.633$ ), waist circumference $\quad(91.34 \pm 17.98 ; \quad 96.50 \pm 16.72 ; \quad 98.18 \pm 17.52$, $P=0.289)$, ALT $(32.40 \pm 8.58 ; 33.90 \pm 8.85 ; 47.30 \pm 32.29$, $P=0.173)$ and AST $(33.23 \pm 6.67 ; 34.03 \pm 6.52 ; 49.10$ $\pm 40.34, P=0.417)$. Patients with NAFLD had significantly higher GGT (78.67 \pm 31.42 vs $38.60+13.26 ; P<0.001)$, HbA1c $(8.61 \pm 1.51 \%$ vs $5.04 \pm 0.80 \% ; \quad P<0.001)$, TC $(6.97 \pm 0.75$ vs $4.56 \pm 0.74 ; P<0.001)$ and TG $(2.78 \pm 0.51$ vs $1.69 \pm 0.4 ; P<0.001)$ than control. However, the difference was not statistically significant between patients with NAFLD and those without NAFLD regarding HbA1c $(8.61 \pm 1.51 \%$ vs $8.38 \pm 1.34 ; P=0.541)$, TC $(6.97$ \pm 0.75 vs $6.94 \pm 0.84 ; P=0.908)$ and TG $(2.78 \pm 0.51$ vs 2.58 $\pm 0.55 ; P<0.001)$. Patients with NAFLD compared to those without NAFLD had longer duration of diabetes $(8.47 \pm 2.98$ vs $5.62 \pm 2.14 ; P<0.001)$, higher GGT level (78.67 \pm 31.42 vs $43.03 \pm 16.19 ; P<0.001)$ and FLI (78.67 \pm 31.42 vs $43.96 \pm 15.51 ; P<0.001)$. In patients with NAFLD mean FIB-4 index $(1.173 \pm 0.65)$, and APRI $(0.47 \pm 0.40)$. Table 1 shows clinical characteristics and laboratory parameters of T2DM patients and control group.

\section{Serum Levels of CypD in the Studied Population}

Serum CypD was significantly higher in T2DM patients compared to control subjects $(11.65 \pm 2.96$ vs $6.58 \pm 1.90 ; P<0.001)$ (Table 1). Moreover, T2DM patients with NAFLD had significantly higher CypD level $(11.65 \pm 2.96 \mathrm{ng} / \mathrm{mL})$ compared to 
Table I Baseline Clinical Parameters of the Study Groups

\begin{tabular}{|c|c|c|c|c|}
\hline Variable & Control & Diabetic Patients without NAFLD & Diabetic Patients with NAFLD & $P$-value \\
\hline Age (years) & $46.60 \pm 9.61$ & $50.10 \pm 8.29$ & $50.10 \pm 8.45$ & 0.212 \\
\hline Duration of diabetes(years) & - & $5.62 \pm 2.14$ & $8.47 \pm 2.98$ & $<0.00 I^{*}$ \\
\hline $\mathrm{BMI}\left(\mathrm{Kg} / \mathrm{m}^{2}\right)$ & $28.66 \pm 4.31$ & $28.48 \pm 5.40$ & $27.51 \pm 5.15$ & 0.633 \\
\hline Waist circumference $(\mathrm{cm})$ & $91.34 \pm 17.98$ & $96.50 \pm 16.72$ & $98.18 \pm 17.52$ & 0.289 \\
\hline Platelet count & $(346 \pm 179) \times 10^{3}$ & $(414 \pm 730) \times 10^{3}$ & $(414 \pm 788) \times 10^{3}$ & $0.047^{*}$ \\
\hline FBG (mmol/L) & $4.55 \pm 0.57$ & $9.9+3.7$ & $10.28+4.19$ & $<0.00 I^{*}$ \\
\hline HbAlc (\%) & $5.04 \pm 0.80$ & $8.38 \pm 1.34$ & $8.61 \pm 1.51$ & $<0.00 I^{*}$ \\
\hline TG (mmol/L) & $1.69 \pm 0.4$ & $2.58 \pm 0.55$ & $2.78+0.51$ & $<0.00$ I* \\
\hline $\mathrm{TC}(\mathrm{mmol} / \mathrm{L})$ & $4.56 \pm 0.74$ & $6.94+0.84$ & $6.97+0.75$ & $<0.00$ I* \\
\hline ALT (IU/L) & $32.40 \pm 8.58$ & $33.90 \pm 8.85$ & $47.30 \pm 32.29$ & 0.173 \\
\hline AST (IU/L) & $33.23 \pm 6.67$ & $34.03 \pm 6.52$ & $49.10 \pm 40.34$ & 0.417 \\
\hline GGT (IU/L) & $38.60 \pm 13.26$ & $43.03 \pm 16.19$ & $78.67 \pm 31.42$ & $<0.001 *$ \\
\hline INR & $1.16 \pm 0.15$ & $1.13 \pm 0.15$ & $1.11 \pm 0.17$ & 0.472 \\
\hline СурD (ng/mL) & $3.5 \mathrm{I} \pm 1.47$ & $6.58 \pm 1.90$ & $11.65 \pm 2.96$ & $<0.001 *$ \\
\hline FLI & - & $43.96 \pm|5.5|$ & $78.24 \pm 18.50$ & $<0.001 *$ \\
\hline FIB-4 index & - & - & $1.173 \pm 0.65$ & - \\
\hline APRI & - & - & $0.47 \pm 0.40$ & - \\
\hline NFS & - & - & $\begin{array}{c}>0.676 \text { (I patient) } \\
<-1.455 \text { (I2 patients) } \\
-1.455-0.676 \text { (I7patients) }\end{array}$ & - \\
\hline
\end{tabular}

Note: *Statistical significance at the $\mathrm{p}<0.05$ level.

Abbreviations: T2DM, type 2 diabetes mellitus; NAFLD, nonalcoholic fatty liver disease; BMI, body mass index; FBG, fasting blood glucose; HbAIc, glycated hemoglobin; TG, triglycerides; TC, total cholesterol; ALT, alanine aminotransferase; AST, aspartate aminotransferase; GGT, gamma-glutamyl transpeptidase; INR, international normalized ratio; CypD, cyclophilin D; FLI, fatty liver index; FIB-4, fibrosis-4 index; APRI; AST to platelet ratio index; NFS, NAFLD fibrosis score.

those without NAFLD $\quad(6.58+1.90 \quad \mathrm{ng} / \mathrm{mL}, \quad P<0.001)$ (Figure 1). Patients NAFLD with FIB-4 $>1.45$ had significantly higher CypD level $(14.27 \pm 2.77 \mathrm{ng} / \mathrm{mL})$ than those with FIB-4 $<1.45(10.70 \pm 2.44 \mathrm{ng} / \mathrm{mL}, P=0.01)$ (Figure 2).

\section{Correlations of Serum CypD and}

\section{Different Variables in Diabetic Patients with NAFLD}

Pearson's correlation was used to assess the correlation between CypD and different variables. There was significant positive correlation between CypD and FIB-4 index $(P=0.001)$, APRI $(P=0.013)$ and NFS $(P<0.001)$. However, there was no significant correlation between CypD level and age $(P=0.877)$, BMI $(P=0.117)$, ALT $(P=0.693)$, HbA1c $(P=0.313)$, total cholesterol $(P=0.888)$ (Table 2$)$.
Uni- and Multivariable Logistic Regression Analyses of Determinants of NAFLD

Using presence or absence of NAFLD as state variable univariate regression analysis revealed a significant positive association between NAFLD and GGT [OR, 1.068 (1.033-1.104); $P<0.001]$, CypD [OR, 2.492 (1.594-3.895); $P<0.001]$ and FLI [OR, 1.131 (1.062-1.204); $P<0.001]$. No association was found between NAFLD and BMI $(P=0.476)$, HbA1C $(P=0.541)$, TC $(P=0.908)$, TG $(P=0.345)$ (Table 3$)$. The association between CypD and NAFLD remained significant when adjusted for GGT and FLI [OR, 1.968 (1594-2.540); $P<0.001]$. In a multivariate logistic regression model, which included variables significantly associated with NAFLD in the univariate analyses, only CypD [OR, 2.027 (1.128-3.642); $P=0.018]$ and GGT [OR, $1.057(1.002-1.116) ; P=0.041]$. 


\section{Cyclophilin D level (ng/mL)}

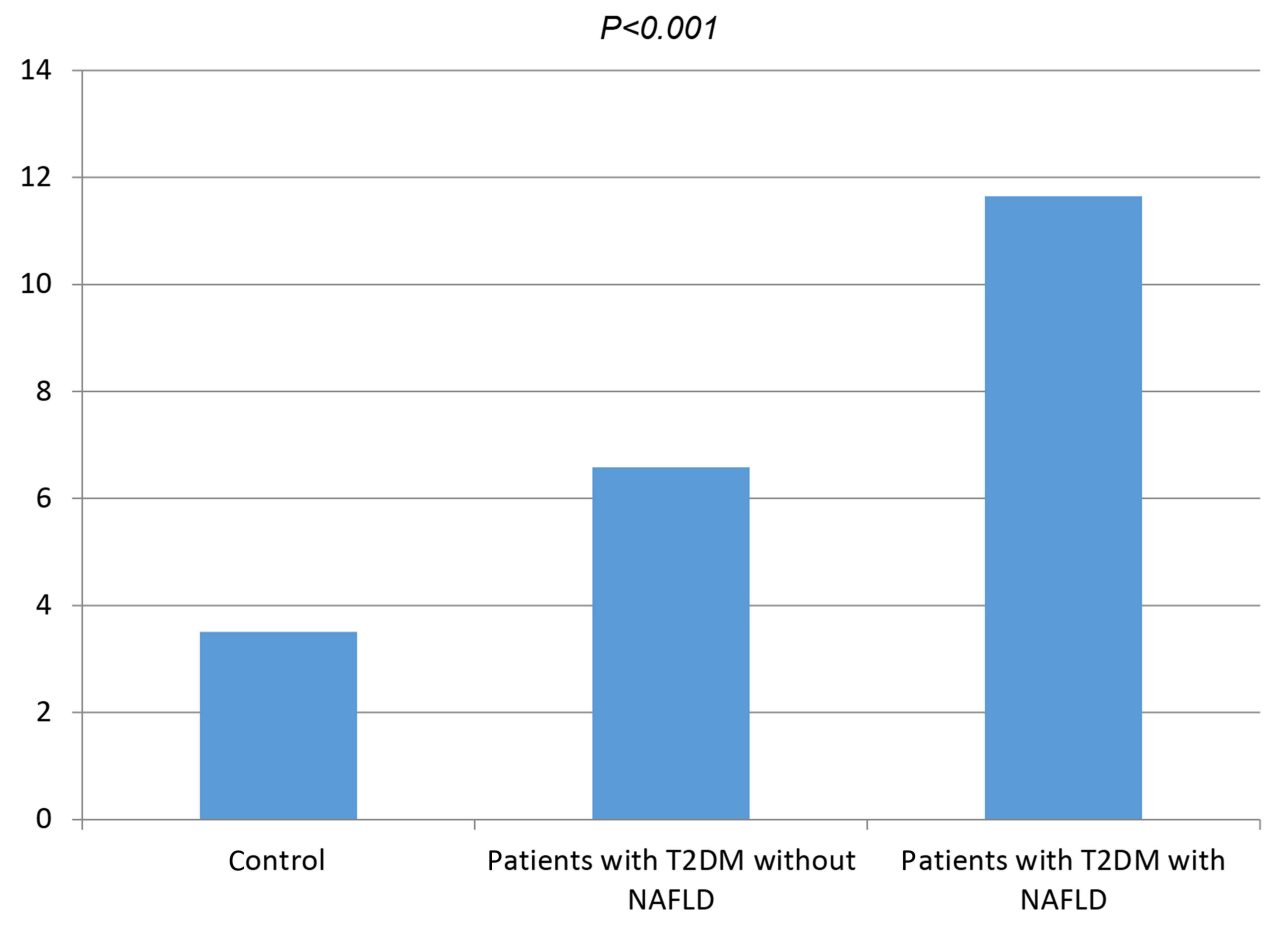

Figure I Serum level of cyclophilin D was significantly higher in T2DM patients without NAFLD compared to control subjects $(6.58 \pm I .90 \mathrm{vs} 3.5 \mathrm{I} \pm \mathrm{I} .47 \mathrm{ng} / \mathrm{mL}$, respectively, $P<0.00 \mathrm{I})$. T2DM patients with NAFLD had significantly higher cyclophilin $D$ level than those without NAFLD $(11.65 \pm 2.96 \mathrm{vs} 6.58 \pm 1.90 \mathrm{ng} / \mathrm{mL}$, respectively, $P<0.00 \mathrm{I})$.

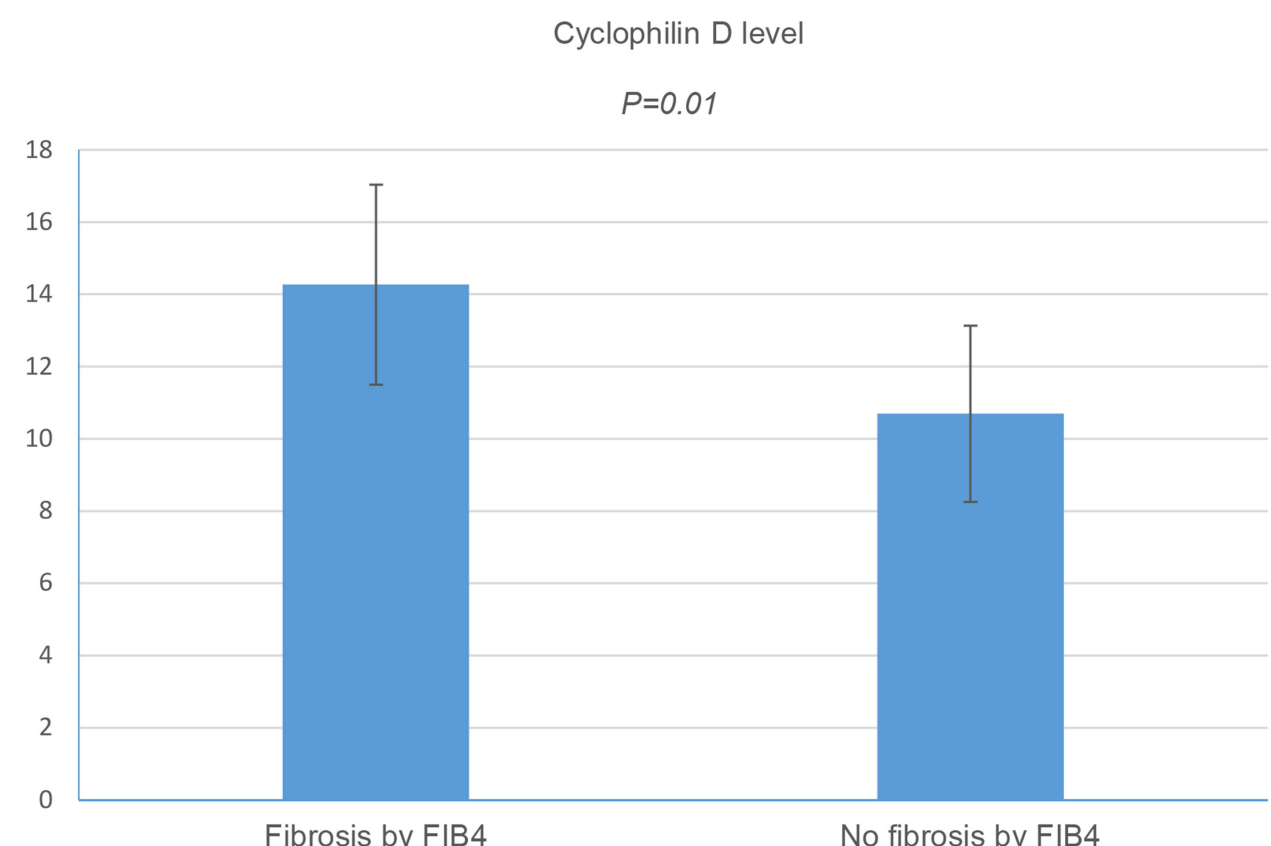

Fibrosis by FIB4

No fibrosis by FIB4

Figure 2 Cyclophilin D was significantly elevated in NAFLD patients with fibrosis as identified by FIB-4 compared with those without fibrosis ( $14.27 \pm 2.77$ vs $10.70+2.44$, respectively, $P=0.010)$. 
Table 2 Correlations of Serum Cyclophilin D with Different Variables in Diabetic Patients with NAFLD

\begin{tabular}{|l|c|c|}
\hline Parameter & $\mathbf{r}$ & $\boldsymbol{P}$-value \\
\hline Age (years) & -0.030 & 0.877 \\
BMI (kg/m ${ }^{2}$ ) & 0.292 & 0.117 \\
Waist circumference (cm) & 0.158 & 0.404 \\
Platelet count & -0.22 & 0.30 \\
FBG (mmol/mol) & -0.144 & 0.447 \\
HbAIC & 0.191 & 0.313 \\
TG (mmol/L) & 0.233 & 0.214 \\
Cholesterol (mmol/L) & -0.027 & 0.888 \\
ALT (IU/L) & 0.084 & 0.693 \\
AST (IU/L) & 0.155 & 0.460 \\
GGT (IU/L) & 0.175 & 0.352 \\
INR & -0.094 & 0.621 \\
FIB-4 index & 0.568 & $0.00 I^{*}$ \\
APRI & 0.446 & $0.013^{*}$ \\
NFS & 0.599 & $<0.00 I^{*}$ \\
\hline
\end{tabular}

Note: *Statistical significance at the $P<0.05$ level.

Abbreviations: BMI, body mass index; FBG, fasting blood glucose; HbAlc, glycated hemoglobin; TG, triglycerides; ALT, alanine aminotransferase; AST, aspartate aminotransferase; GGT, gamma-glutamyl transpeptidase; INR, international normalized ratio; FIB-4, fibrosis-4; APRI, AST to platelet ratio index; NFS, NAFLD fibrosis score.

\section{Uni- and Multivariable Logistic Regression Analyses of Determinants of Liver Fibrosis in Patients with NAFLD}

Patients with NAFLD were divided into two groups based on FIB-4 index: patients with FIB-4 $>1.45$ (patients with fibrosis) and patients with FIB-4 $<1.45$ (patients with no fibrosis). CypD [OR, 1.687 (1.130-2.517); $P=0.010$ ], age [OR, 1.142 (1.005-1.298); $P=0.042]$ were independent prognostic factors of liver fibrosis in univariate analysis (Table 4). In multivariable analysis CypD was significant independent predictor for liver fibrosis [OR, 1.992 (1.1713.391); $P=0.011]$

\section{CypD as Predictor of Fatty Liver and Liver Fibrosis}

ROC curve was calculated on the basis of CypD level and FLI using NAFLD presence or absence as state variable. CypD had higher sensitivity but lower specificity than FLI in diagnosis of NAFLD. The AUC was 0.922 (95\% confidence interval (CI); 0.853-0.992, $P<0.001)$ with the best cutoff value of CypD was 7.55 (Figure 3).

ROC curves of CypD, APRI and NFS for detecting fibrosis as defined by FIB-4 showed that the AUROC was highest for NFS at 0.989 , followed by the CypD (AUROC $=0.835$ ) at $14.055 \mathrm{ng} / \mathrm{mL}$ and APRI (AUROC $=0.886$ ) at 0.339 . The sensitivity of $\mathrm{CypD}$ $(75 \%)$ as a predictor of liver fibrosis was lower than APRI (87.5\%) and NFS (100\%). However, the specificity of CypD (81.8\%) in prediction of hepatic fibrosis was higher than APRI (77.3\%) but, lower than NFS (95.5\%) (Figure 4).

\section{Discussion}

CypD is a main regulator of the mitochondrial permeability, and it is localized in the mitochondria. ${ }^{21}$ A growing body of evidence points to the role of mitochondrial dysfunction and disorder of mitophagy homeostasis in the

Table 3 Univariate Analyses of Determinants of NAFLD

\begin{tabular}{|c|c|c|c|c|c|c|}
\hline \multirow[t]{2}{*}{ Variable } & \multirow{2}{*}{ NAFLD (Mean \pm SD) } & \multirow[t]{2}{*}{ No NAFLD (Mean \pm SD) } & \multirow[t]{2}{*}{$P$-value } & \multirow[t]{2}{*}{ OR } & \multicolumn{2}{|c|}{$95 \% \mathrm{Cl}$} \\
\hline & & & & & Lower & Upper \\
\hline FBG (mg/dl) & $9.9 \pm 3.7$ & $10.28 \pm 4.19$ & 0.711 & 0.999 & 0.991 & 1.006 \\
\hline HbAlc (\%) & $8.61 \pm 1.51$ & $8.38 \pm 1.34$ & $0.54 I$ & 1.119 & 0.780 & 1.607 \\
\hline ALT (IU/L) & $47.30 \pm 32.29$ & $33.90 \pm 8.85$ & 0.074 & 1.035 & 0.997 & 1.074 \\
\hline AST (IU/L) & $49.10 \pm 40.34$ & $34.03 \pm 6.52$ & 0.103 & 1.034 & 0.993 & 1.077 \\
\hline Platelet count & $414 \pm 78.8 \times 10^{3}$ & $414 \pm 73 \times 10^{3}$ & 0.994 & 1.000 & 1.000 & 1.000 \\
\hline GGT (IU/L) & $78.67 \pm 31.42$ & $41.37 \pm 16.30$ & $<0.001 *$ & 1.068 & 1.033 & 1.104 \\
\hline TG $(\mathrm{mmol} / \mathrm{L})$ & $2.58 \pm 0.55$ & $2.78 \pm 0.51$ & 0.527 & 1.003 & 0.994 & 1.012 \\
\hline $\mathrm{TC}(\mathrm{mmol} / \mathrm{L})$ & $6.94 \pm 0.84$ & $6.97 \pm 0.75$ & 0.908 & 0.999 & 0.982 & 1.016 \\
\hline Age (years) & $50.10 \pm 8.45$ & $50.10 \pm 8.29$ & 1.000 & 1.000 & 0.940 & 1.063 \\
\hline BMI $\left(\mathrm{kg} / \mathrm{m}^{2}\right)$ & $27.51 \pm 5.15$ & $28.48 \pm 5.40$ & 0.476 & 0.965 & 0.875 & 1.065 \\
\hline Waist (cm) & $98.18 \pm 17.52$ & $96.50 \pm 16.72$ & 0.699 & 1.006 & 0.976 & 1.037 \\
\hline СурD (ng/mL) & $11.65 \pm 2.96$ & $6.58 \pm 1.90$ & $<0.001 *$ & 2.492 & 1.594 & 3.895 \\
\hline FLI & $78.24 \pm 18.50$ & $48.08 \pm 11.97$ & $<0.00 I^{*}$ & 1.131 & 1.062 & 1.204 \\
\hline
\end{tabular}

Note: *Statistical significance at the $P<0.05$ level.

Abbreviations: NAFLD, nonalcoholic fatty liver disease; FBG, fasting blood glucose; HbAlc, glycated hemoglobin; ALT, alanine aminotransferase; AST, aspartate aminotransferase; GGT, gamma-glutamyl transpeptidase; TG, triglycerides; TC, total cholesterol; BMI, body mass index; CypD, cyclophilin D; FLI, fatty liver index. 
Table 4 Univariate Logistic Regression for Predictors of Fibrosis in Patients with NAFLD

\begin{tabular}{|c|c|c|c|c|c|c|}
\hline \multirow[t]{2}{*}{ Variable } & \multirow{2}{*}{$\begin{array}{c}\text { Fibrosis by FIB- } \\
4 \text { (Mean } \pm S D \text { ) }\end{array}$} & \multirow{2}{*}{$\begin{array}{c}\text { No fibrosis by } \\
\text { FIB-4 (Mean } \pm \text { SD) }\end{array}$} & \multirow[t]{2}{*}{$P$-value } & \multirow[t]{2}{*}{ OR } & \multicolumn{2}{|c|}{$95 \% \mathrm{Cl}$} \\
\hline & & & & & Lower & Upper \\
\hline $\mathrm{FBG}(\mathrm{mmol} / \mathrm{mol})$ & $8.4 I \pm 3.14$ & $10.46 \pm 3.79$ & 0.184 & 0.990 & 0.977 & 1.005 \\
\hline HbAlc (\%) & $8.36 \pm 1.05$ & $8.70 \pm 1.66$ & 0.590 & 0.855 & 0.484 & 1.511 \\
\hline ALT (IU/L) & $67.38 \pm 50.37$ & $40.00 \pm \mid 9.61$ & 0.064 & 1.025 & 0.999 & 1.052 \\
\hline AST (IU/L) & $72.38 \pm 66.63$ & $40.64 \pm 22.05$ & 0.106 & 1.019 & 0.996 & 1.043 \\
\hline Platelet count & $385 \pm 91.2 \times 10^{3}$ & $425 \pm 73.3 \times 10^{3}$ & 0.225 & 1.000 & 1.000 & 1.000 \\
\hline GGT (IU/L) & $84.38 \pm 40.78$ & $76.59 \pm 28.14$ & 0.545 & 1.008 & 0.982 & 1.035 \\
\hline TG (mmol/L) & $2.74 \pm 0.64$ & $2.53 \pm 0.52$ & 0.345 & 1.008 & 0.991 & 1.025 \\
\hline $\mathrm{TC}(\mathrm{mmol} / \mathrm{L})$ & $6.84 \pm 1.06$ & $6.98 \pm 0.78$ & 0.690 & 0.995 & 0.970 & 1.020 \\
\hline Age (years) & $55.63 \pm 5.18$ & $48.09 \pm 8.59$ & $0.042 *$ & 1.142 & 1.005 & 1.298 \\
\hline BMI $\left(\mathrm{kg} / \mathrm{m}^{2}\right)$ & $30.13 \pm 6.86$ & $26.56 \pm 4.17$ & 0.106 & 1.150 & 0.971 & 1.361 \\
\hline Waist (cm) & $95.25 \pm 19.88$ & $99.25 \pm 16.96$ & 0.576 & 0.987 & 0.942 & 1.034 \\
\hline СурD (ng/mL) & $\mid 4.27 \pm 2.77$ & $10.70 \pm 2.44$ & $0.010 *$ & 1.687 & 1.130 & 2.517 \\
\hline FLI & $79.60 \pm 17.39$ & $77.75 \pm 19.26$ & 0.805 & 1.006 & 0.960 & 1.054 \\
\hline APRI & $0.93 \pm 0.60$ & $0.30 \pm 0.10$ & $0.040 *$ & 2820.770 & 1.462 & $5,440,783.742$ \\
\hline NFS & $0.12 \pm 0.6 \mathrm{I}$ & $-2.03- \pm 0.74$ & 0.165 & 8430.318 & 0.024 & $2,949,835,693.943$ \\
\hline
\end{tabular}

Note: *Statistical significance at the $P<0.05$ level.

Abbreviations: NAFLD, nonalcoholic fatty liver disease; FBG, fasting blood glucose; HbAlc, glycated hemoglobin; ALT, alanine aminotransferase; AST, aspartate aminotransferase; GGT, gamma-glutamyl transpeptidase; TG, triglycerides; TC, total cholesterol; BMI, body mass index; CypD, cyclophilin D; FLI, fatty liver index; FIB-4, fibrosis-4; APRI, AST to platelet ratio index; NFS, NAFLD fibrosis score.

development of insulin resistance, metabolic derangement and hepatic steatosis and fibrosis. ${ }^{22,23,24}$ However, most of it came from investigations on the cellular and molecular levels. $^{12,14,22}$ Recently, Alfonso et al reported a significant association between serum level of CypC, CypA, and CypB, but not CypD and coronary artery disease and several metabolic parameters. ${ }^{25}$ Also, Mutlu et al found that serum levels of CypA are elevated in patients with NAFLD, particularly those with diabetes. ${ }^{26}$ Whether serum CypD could be a surrogate marker of hepatic expression of this ubiquitous protein needs to be addressed. The main finding of the current study was significant elevation of serum CypD in T2DM patients with NAFLD and CypD level more than $7.55(\mathrm{ng} / \mathrm{mL})$ was able to identify diabetic patients with NAFLD with a higher sensitivity than FLI. Furthermore, there was statistically significant correlation of CypD and different fibrosis scores in patients with NAFLD.

Various experimental studies have shown the role of CypD in the development of hepatic steatosis. ${ }^{12,14,26,27}$ In mice fed a high-fat diet, CypD expression increased and preceded the accumulation of hepatic triglycerides and the development of hepatic steatosis. ${ }^{27}$ Moreover, in mice administered a normal diet overexpression of CypD induced hepatic steatosis and hepatic TG accumulation without change in serum level of fatty acids or TG suggesting a direct causal relationship between CypD and NAFLD. ${ }^{14,27}$ In contrast, CypD deletion decreased or totally prevented steatosis and weight gain in mice following a high fat diet. ${ }^{26-28}$

Liver fibrosis invariably results from persistent hepatocyte injury and inflammation, and halting fibrosis is a major target in treating nonalcoholic steatohepatitis (NASH). CypD was found to play a principle role in the fibrotic process in multiple parenchymal organs such as kidney, lung, and heart. ${ }^{13,29}$ We observed that CypD levels were significantly increased in NAFLD patients with hepatic fibrosis as defined by FIB-4 than those without fibrosis. Moreover, we found significant positive correlation between serum level CypD and NFS and APRI in patients with NAFLD.

A number of studies investigated the relation between CypD and liver fibrosis. ${ }^{30-32}$ Suppression both of CypDinduced mitochondrial permeability transition and CypA and B-associated inflammatory events inhibited the development of cirrhosis in animal models of liver fibrosis. ${ }^{30}$ Another study found that inhibition of CypB and $\mathrm{D}$ reduced collagen type III and attenuated liver fibrosis in a CCl4-induced rat liver fibrosis model. ${ }^{31}$ Furthermore, CRV431 which is a nonselective cyclophilin-A, B, D, and $\mathrm{G}$ inhibitor decreased liver fibrosis in a mouse model of $\mathrm{NASH}^{32}$ 


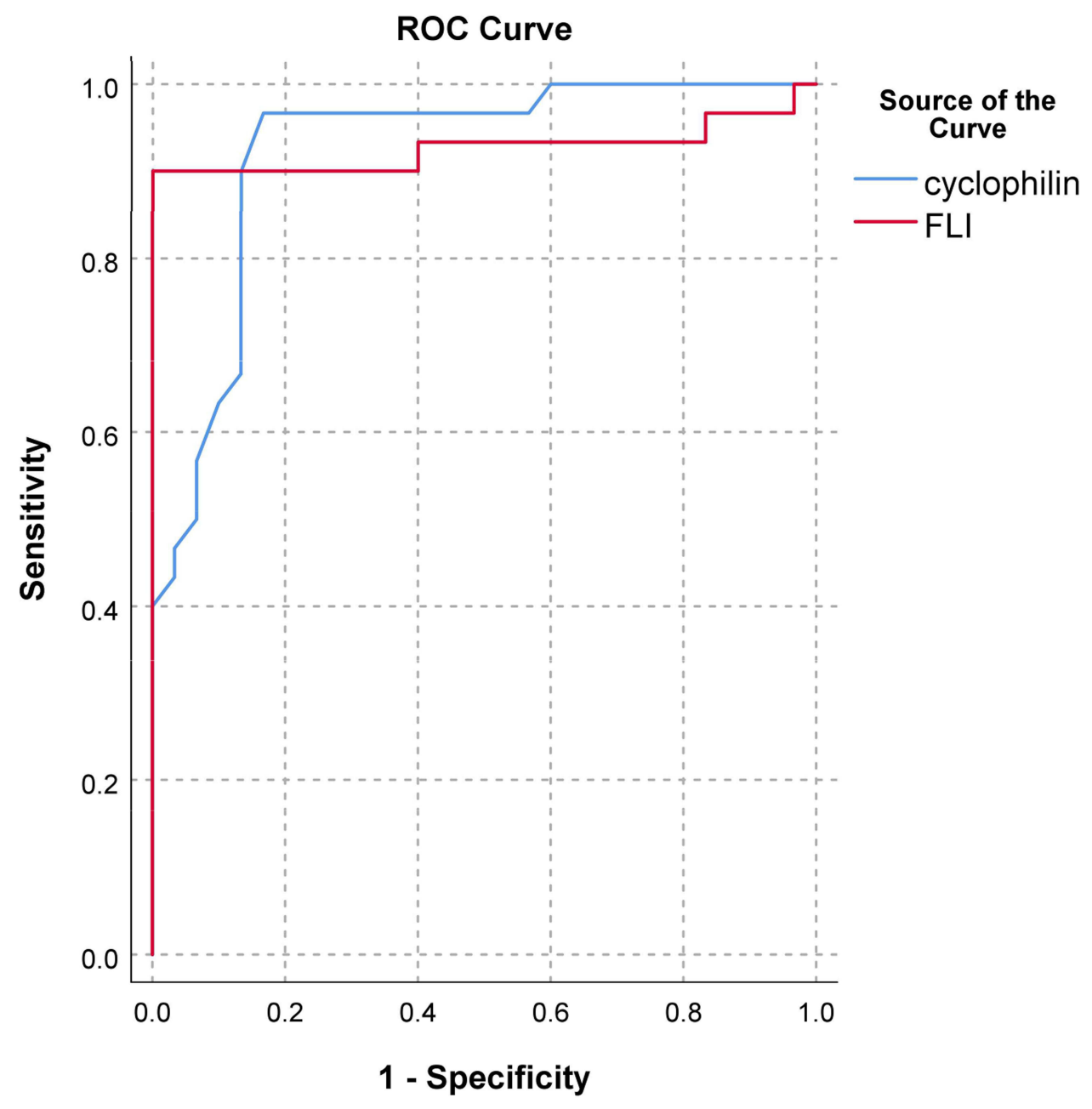

Figure 3 Receiver-operating-characteristic curves (ROC) describing cyclophilin D (CyPD) as predictor of nonalcoholic fatty liver disease compared to FLI in patients with diabetes. CypD, the AUC was 0.922 ( $95 \%$ confidence interval (Cl):, $0.853-0.992, P<.001$ ), cut-ff: $7.55 \mathrm{ng} / \mathrm{mL}$, sensitivity: $96.7 \%$, specificity: $83.3 \%$. FLI, the AUC was 0.927 ( $95 \% \mathrm{Cl}$ : $0.842-1.01 \mathrm{I}, P<0.001$ ), cutoff value: 62.50 , sensitivity: $60 \%$, specificity: $100 \%$.

Although there are many good noninvasive tools to assess fibrosis in patients with NAFLD, continued research is needed to identify more markers that evaluate the different molecular mechanisms involved in liver fibrosis. In the present study, serum CypD was a significant marker in identifying patients with higher fibrosis scores with CypD level more than $14.05(\mathrm{ng} / \mathrm{mL})$ had $75 \%$ sensitivity and $81.1 \%$ specificity in prediction of hepatic fibrosis. Although, we found serum CypD was a more specific predictor of liver fibrosis than APRI, serum CypD was less sensitive than APRI and NFS.

In our study although serum CypD levels were higher in patients with diabetes than the healthy control group, we failed to demonstrate a significant correlation between CypD and blood glucose. Moreover, the relation of CypD and NAFLD and fibrosis scores was independent from blood glucose, lipid and BMI. Previous studies showed that
CypD levels are elevated in animal models of obesity and metabolic syndrome and reduce when the disease is reversed. ${ }^{13,14,33}$ Similar to our results, a recent study of patients with coronary artery disease found no correlation between serum CypD and blood glucose, cholesterol, or $\mathrm{TG}^{25}$ Recent study showed that CypD promotes nonglucose-stimulated insulin secretion, but not glucose-stimulated insulin secretion, through enhancing mitochondrial proton leak which could explain the absence of significant correlation between Hbalc and CypD in our study. ${ }^{34}$ Another study reported a significant correlation between the serum CypA and blood glucose levels, but there was no significant correlation with other metabolic parameters in patients with NAFLD. ${ }^{26}$ Furthermore, CypD has a tissue-specific effect in the metabolic homeostasis that the liver mitochondria are more dependent on CypD for mPTP opening than skeletal muscle mitochondria. ${ }^{12}$ The data in this study could point to 


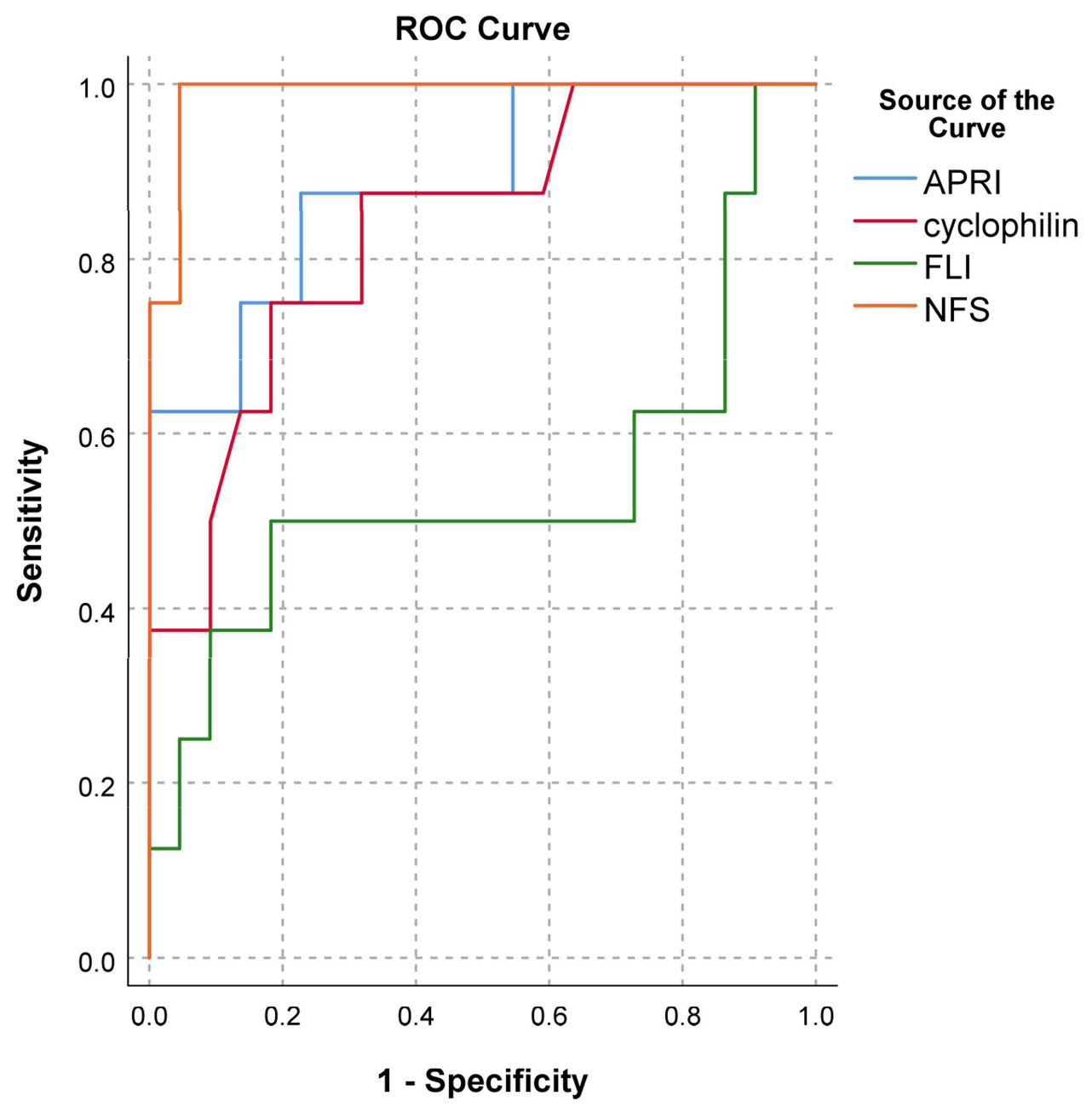

Figure 4 Receiver-operating-characteristic curves (ROC) describing cyclophilin D (CyPD) as predictor of liver fibrosis as identified by FIB-4 $>$ I.45 compared to AST to platelet ratio index (APRI) and NAFLD fibrosis score (NFS) in patients with nonalcoholic fatty liver disease. CypD, AUROC=0.835 ( $95 \% \mathrm{Cl}: 0.672-0.998, P<0.00 \mathrm{I})$, cutoff $=$ $14.05 \mathrm{ng} / \mathrm{mL}$, specificity $=81.1 \%$, sensitivity $=75 \%$. APRI, AUROC $=0.886(95 \% \mathrm{Cl}: 0.745-1.028, P<0.001)$, cutoff $=0.339$, specificity=77.3\%, sensitivity $=87$. $\%$. NFS, AUROC $=0.989(95 \% \mathrm{Cl}: 0.960-1.018, P<0.001)$, cutoff $=-0.710$, specificity $=95.5 \%$, sensitivity $=100 \%$.

a direct effect of CypD in the development of liver steatosis and fibrosis irrespective of change of systemic metabolism.

Some limitations can be discussed in the present study. First, ultrasound was used to identify patients with NAFLD which is an operator-dependent technique. However, we used previously validated diagnostic criteria and the examination was performed by one sonographer. In addition, we calculated FLI for all patients. Second, noninvasive scores were used to define patients with fibrosis. Although liver biopsy is the most accurate method in diagnosis of NAFLD and liver fibrosis; it is an invasive technique with possible serious complications. Other noninvasive imaging techniques that measure liver stiffness such as elastography could not be used because of the cost. Nevertheless, we calculated multiple fibrosis scores that correlate well with the results of liver biopsy. Third, CypD primarily exerts its action at mitochondrial level and there is no data about the serum levels of CypD as a surrogate of hepatic expression. However, CypD can be measured in serum and we found a significant difference in serum levels of CypD in healthy subjects and patients with and without NAFLD. Fourth, the relatively small number of the studied population; a larger study is needed to confirm the results.

\section{Conclusion}

This was the first study that assessed the serum CypD in diabetic patients with NAFLD and we found that serum CypD levels were higher in diabetic patients with NAFLD than those without NAFLD. In addition, CypD was a highly sensitive predictor of NAFLD. Furthermore, CypD had significant correlation with different fibrosis scores in diabetic patients with NAFLD irrespective of 
the other metabolic parameters. Consequently, CypD can be used as a noninvasive marker for diagnosis and assessing the severity of NAFLD. Also, CypD may serve as a potential novel therapeutic strategy for NAFLD, and CypD inhibitors could decrease liver fibrosis in patients with NAFLD.

\section{Acknowledgment}

All authors acknowledge their gratitude to the staff members of diabetes and endocrine clinic for their help and support.

\section{Funding}

The authors received no specific funding for this work.

\section{Disclosure}

The authors report no conflicts of interest in this work.

\section{References}

1. Mitra S, De A, Chowdhury A. Epidemiology of non-alcoholic and alcoholic fatty liver diseases. Transl Gastroenterol Hepatol. 2020;5:16. doi:10.21037/tgh.2019.09.08

2. Buzzetti E, Pinzani M, Tsochatzis EA. The multiple-hit pathogenesis of non-alcoholic fatty liver disease (NAFLD). Metabolism. 2016;65 (8):1038-1048. doi:10.1016/j.metabol.2015.12.012

3. Pappachan JM, Babu S, Krishnan B, Ravindran NC. Non-alcoholic fatty liver disease: a clinical update. J Clin Transl Hepatol. 2017;5 (4):384-393. doi:10.14218/JCTH.2017.00013

4. Bataller R, Rombouts K, Altamirano J, Marra F. Fibrosis in alcoholic and nonalcoholic steatohepatitis. Best Pract Res Clin Gastroenterol. 2011;25(2):231-244. doi:10.1016/j.bpg.2011.02.010

5. Parthasarathy G, Revelo X, Malhi H. Pathogenesis of nonalcoholic steatohepatitis: an overview. Hepatol Commun. 2020;4(4):478-492. doi:10.1002/hep4.1479 PMID: 32258944; PMCID: PMC7109346.

6. Haas JT, Francque S, Staels B. Pathophysiology and mechanisms of nonalcoholic fatty liver disease. Annu Rev Physiol. 2016;78 (1):181-205. PMID: 26667070. doi:10.1146/annurev-physiol $-021115-105331$

7. Rieusset J, Fauconnier J, Paillard M, et al. Disruption of calcium transfer from ER to mitochondria links alterations of mitochondria-associated ER membrane integrity to hepatic insulin resistance. Diabetologia. 2016;59(3):614-623. PMID: 26660890. doi: 10.1007/s00125-015-3829-8

8. Mansouri A, Gattolliat $\mathrm{CH}$, Asselah T. Mitochondrial dysfunction and signaling in chronic liver diseases. Gastroenterology. 2018;155 (3):629-647. PMID: 30012333. doi:10.1053/j.gastro.2018.06.083

9. Zorov DB, Juhaszova M, Yaniv Y, Nuss HB, Wang S, Sollott SJ. Regulation and pharmacology of the mitochondrial permeability transition pore. Cardiovasc Res. 2009;83(2):213-225. PMID: 19447775; PMCID: PMC2701724. doi:10.1093/cvr/cvp151

10. Giorgi C, Agnoletto C, Bononi A, et al. Mitochondrial calcium homeostasis as potential target for mitochondrial medicine. Mitochondrion. 2012;12(1):77-85. PMID: 21798374; PMCID: PMC3281195. doi: 10.1016/j.mito.2011.07.004

11. Wacquier B, Combettes L, Dupont G. Dual dynamics of mitochondrial permeability transition pore opening. Sci Rep. 2020;10(1):3924. PMID: 32127570; PMCID: PMC7054270. doi:10.1038/s41598-02060177-1.
12. Laker RC, Taddeo EP, Akhtar YN, Zhang M, Hoehn KL, Yan Z. The mitochondrial permeability transition pore regulator cyclophilin D exhibits tissue-specific control of metabolic homeostasis. PLoS One. 2016;11(12):e0167910. doi:10.1371/journal.pone.0167910

13. Porter GA Jr, Beutner G. Cyclophilin D, somehow a master regulator of mitochondrial function. Biomolecules. 2018;8(4):176. doi:10.3390/ biom8040176

14. Wang X, Du H, Shao S, et al. Cyclophilin D deficiency attenuates mitochondrial perturbation and ameliorates hepatic steatosis. Hepatology. 2018;68(1):62-77. doi:10.1002/hep.29788

15. American Diabetes Association. Diagnosis and classification of diabetes mellitus. Diabetes Care. 2010;33 Suppl 1(Suppl 1):S62-S69. doi:10.2337/dc10-S062.

16. Palmentieri B, de Sio I, La Mura V, et al. The role of bright liver echo pattern on ultrasound B-mode examination in the diagnosis of liver steatosis. Dig Liver Dis. 2006;38(7):485-489. doi:10.1016/j. dld.2006.03.021

17. Bedogni G, Bellentani S, Miglioli L, et al. The fatty liver index: a simple and accurate predictor of hepatic steatosis in the general population. BMC Gastroenterol. 2006;6(1):33. doi:10.1186/1471230X-6-33

18. Sun W, Cui H, Li N, et al. Comparison of FIB-4 index, NAFLD fibrosis score and BARD score for prediction of advanced fibrosis in adult patients with non-alcoholic fatty liver disease: a meta-analysis study. Hepatol Res. 2016;46(9):862-870. doi:10.1111/hepr.12647

19. Wai CT, Greenson JK, Fontana RJ, et al. A simple noninvasive index can predict both significant fibrosis and cirrhosis in patients with chronic hepatitis C. Hepatology. 2003;38(2):518-526. doi:10.1053/ jhep.2003.50346

20. Angulo P, Hui JM, Marchesini G, et al. The NAFLD fibrosis score: a noninvasive system that identifies liver fibrosis in patients with NAFLD. Hepatology. 2007;45(4):846-854. doi:10.1002/hep.21496

21. Naoumov NV. Cyclophilin inhibition as potential therapy for liver diseases. $J$ Hepatol. 2014;61(5):1166-1174. doi:10.1016/j. jhep.2014.07.00

22. Zhou H, Zhu P, Wang J, Toan S, Ren J. DNA-PKcs promotes alcohol-related liver disease by activating Drp1-related mitochondrial fission and repressing FUNDC1-required mitophagy. Signal Transduct Target Ther. 2019;4(1):56. doi:10.1038/s41392-019-0094-1

23. Gao A, Jiang J, Xie F, Chen L. Bnip3 in mitophagy: novel insights and potential therapeutic target for diseases of secondary mitochondrial dysfunction. Clin Chim Acta. 2020;506:72-83. doi:10.1016/j. cca.2020.02.024

24. Tavecchio M, Lisanti S, Bennett MJ, Languino LR, Altieri DC. Deletion of cyclophilin D impairs $\beta$-oxidation and promotes glucose metabolism. Sci Rep. 2015;5(1):15981. PMID: 26515038; PMCID: PMC4626838. doi:10.1038/srep15981

25. Alfonso A, Bayón J, Gegunde S, et al. High Serum cyclophilin $\mathrm{C}$ levels as a risk factor marker for coronary artery disease. Sci Rep. 2019;9(1):10576. doi:10.1038/s41598-019-46988-x

26. Mutlu HH, Caklili OT, Coskunpinar E. Serum concentrations of cyclophilin A in patients with nonalcoholic fatty liver disease. Acta Gastroenterol Belg. 2017;80(1):3-7.

27. Su X, Lin D, Luo D, et al. Cyclophilin D participates in the inhibitory effect of high-fat diet on the expression of steroidogenic acute regulatory protein. J Cell Mol Med. 2019;23(10):6859-6871. PMID: 31373170; PMCID: PMC6787510. doi: 10.1111/jcmm. 14569

28. Tubbs E, Theurey P, Vial G, et al. Mitochondria-associated endoplasmic reticulum membrane (MAM) integrity is required for insulin signaling and is implicated in hepatic insulin resistance. Diabetes. 2014;63(10):3279-3294. PMID: 24947355. doi: 10.2337/db13-1751

29. Hou W, Leong KG, Ozols E, Tesch GH, Nikolic-Paterson DJ, Ma FY. Cyclophilin D promotes tubular cell damage and the development of interstitial fibrosis in the obstructed kidney. Clin Exp Pharmacol Physiol. 2018;45(3):250-260. PMID: 29230844. doi:10.1111/14401681.12881 
30. Ure DR, Trepanier DJ, Mayo PR, Foster RT. Cyclophilin inhibition as a potential treatment for nonalcoholic steatohepatitis (NASH). Expert Opin Investig Drugs. 2020;29(2):163-178. PMID: 31868526. doi:10.1080/13543784.2020.1703948

31. Wang H, Zhang Y, Wang T, You H, Jia J. N-methyl-4-isoleucine cyclosporine attenuates $\mathrm{CCl}$-induced liver fibrosis in rats by interacting with cyclophilin B and D. J Gastroenterol Hepatol. 2011;26(3):558-567. PMID: 21332552. doi:10.1111/j.1440-1746.2010.06406.x

32. Kuo J, Bobardt M, Chatterji U, et al. A pan-cyclophilin inhibitor, CRV431, decreases fibrosis and tumor development in chronic liver disease models. J Pharmacol Exp Ther. 2019;371(2):231-241. PMID: 31406003; PMCID: PMC6815936. doi: 10.1124/jpet.119.261099
33. Jiang M, Wang C, Meng Q, et al. Cyclosporin A attenuates weight gain and improves glucose tolerance in diet-induced obese mice. Mol Cell Endocrinol. 2013;370(1-2):96-102. PMID: 23499865. doi: 10.1016/j.mce.2013.02.018

34. Taddeo EP, Alsabeeh N, Baghdasarian S, et al. Mitochondrial proton leak regulated by cyclophilin D elevates insulin secretion in islets at nonstimulatory glucose levels. Diabetes. 2020;69(2):131-145. doi:10.2337/db19-0379

\section{Publish your work in this journal}

The International Journal of General Medicine is an international, peer-reviewed open-access journal that focuses on general and internal medicine, pathogenesis, epidemiology, diagnosis, monitoring and treatment protocols. The journal is characterized by the rapid reporting of reviews, original research and clinical studies across all disease areas. The manuscript management system is completely online and includes a very quick and fair peer-review system, which is all easy to use. Visit http://www.dovepress.com/ testimonials.php to read real quotes from published authors.

Submit your manuscript here: https://www.dovepress.com/international-journal-of-general-medicine-journal 